\title{
Influence of BMI on foot posture alignment in resting gait of secondary school children in Lagos metropolis aged 10 to 18 years
}

\author{
Godwin O Mbaka, Mario Adelaja \\ Department of Anatomy, Lagos State University College of Medicine, Ikeja, Nigeria
}

\begin{abstract}
The study was to determine the influence of BMI on foot posture alignment in the resting gait of secondary school children. A localized, two-stage, stratified, cross-sectional, and descriptive survey was carried out in 10 secondary schools involving a sample secondary student population of 518 students, 235 males, and 283 females. The result shows that there was a statistically significant association $\left(\mathrm{X}^{2}=32.4345, \mathrm{p}=0.000\right)$ between the BMI of student's
\end{abstract}

Correspondence: Godwin O. Mbaka, Department of Anatomy, Lagos State University College of Medicine, 1-2 Oba Akinjobi Way, Nigeria. E-mail: mbaakagm@yahoo.com

Key words: Body mass index; foot posture alignment; resting gait; school children.

Contribution: This work was carried out in collaboration between all authors. Author GOM undertook the literature searches, the write-up and final editing of the manuscript as well as partook in the statistical analysis. Author MAA came up with the experimental concept, took part in the write-up and statistical analysis. All authors read and approved the final manuscript.

Conflict of interests: The authors have no conflict of interest to declare.

Availability of data and materials: All data generated or analyzed during this study are included in this published article.

Ethics approval and consent to participate: The study protocol was approved by the Lagos State University Health Research Ethics Committee (LRC/ 06/10/1438). Written permission to conduct the study was obtained from the Lagos State Government through the Ministry of Education District VI, Ideal Primary School, C/O, Apapa-Oshodi Express Way, Lagos.

Informed consent: Written informed consent/assent was obtained having provided necessary identification and study documentation to each school for students, parents, and school administration before the enrolment of their students to participate in the study.

Received for publication: 10 February 2021.

Revision received: 17 June 2021.

Accepted for publication: 17 June 2021.

This work is licensed under a Creative Commons Attribution NonCommercial 4.0 License (CC BY-NC 4.0).

CC Copyright: the Author(s),2021

Licensee PAGEPress, Italy

Annals of African Medical Research 2021; 4:144

doi:10.4081/aamr.2021.144 cohort and their age. There was also a statistically significant association between different age groups and gender at $p<0.046$. Using the statistics of Pearson chi-square analysis, age-dependent variability of BMI $\left(\mathrm{X}^{2}=32.4345, \mathrm{p}=0.000\right)$ was established amongst the study population. The BMI of students for the varying foot alignment was not normally distributed, instead, it was skewed to the right. According to the Kruskal-Wallis H test, there was no statistically significant difference in BMI between the three age cohorts of student's foot alignment in resting gait; Pearson chisquare, $X^{2}(2)=4.575, p=0.1015$. The distribution of the left foot posture alignment according to the FPI scale indicates that overall, $82.13 \%$ of males and $84.40 \%$ of females showed normal foot alignment whereas $10.64 \%$ and $9.22 \%$ of male and female students respectively confirmed slight foot pronation while $7.23 \%$ of male students and $6.38 \%$ of female students showed slightly supinated feet. This study shows that BMI has no influence on FPI in resting gait. The high incidence of underweight amongst the students' population could be a reflection of the low socioeconomic status of many families.

\section{Introduction}

Excessive body weight is exemplified by an increase in Body Mass Index (BMI). In adolescents where morphological changes are more apparent, an excessive weight thrust on the lower extremities would accordingly bring to bear structural changes in the foot position. When this persists into adulthood, it leads to gait problems that usually bring about deformities. ${ }^{1}$ An elevated BMI level has been observed as a key and identifiable risk factor that may influence the core stability and Foot Posture Alignment (FPA). ${ }^{2}$

It has been established that weight-bearing like in obese cases exert significant changes in the foot anatomy because of the increased biomechanical loading which causes alterations in the FPA. ${ }^{3-5}$ BMI is used to determine the prevalence of overweight as shown by documentation of several Nigerian authors amongst diverse population range. ${ }^{6-9}$ Being classified as overweight is considered a predictor for posture stability while a high BMI is seen as an important risk factor for geriatric falling. ${ }^{10}$ The most profound effect of overweight on the foot outside the bony frame stress is the arch collapse that occurs over time. ${ }^{11,12}$ The occurrence of arch collapse would more often result in foot pronation in which case the heels are inclined outward while the ankles become projected inward. ${ }^{13}$

The relationship between BMI and FPA remains contentious although proponent against seems to be holding sway. ${ }^{14,15}$ However, the focus here is on the environmental impact factor. The impact of urban residency is a major factor that influences wellbeing and socio-economic changes which inadvertently affects lifestyle that brings to bear changes in body structure. It is understood that BMI in children and adolescents is affected by environmental factors, such as unhealthy diet, sedentary leisure time, and 
physical inactivity. ${ }^{16}$ On the other hand, the environment has equally been observed to affect foot posture due to its influence on body size. ${ }^{17}$ It has been reported that the human foot is the part of body structure mainly affected by anatomical variations in the entire human body. ${ }^{18}$ The purpose of this study, therefore, was to determine the influence of BMI on foot posture alignment in resting gait of secondary school children in Lagos metropolis with the view to ascertaining environmental impact factor on body size and its cumulative effect on FPA. It is equally intended to investigate FPA distribution amongst the two genders and to also establish the probable linkage of posture alignment in the two legs.

\section{Materials and Methods}

A localized, two-stage, stratified, cross-sectional, and descriptive survey was carried out in 10 secondary schools involving a sample secondary student population of 518 Nigerian adolescents were taken of both male and female respondents across different ethnic groups aged between 10 and 18 years. A total of 235 male students representing $45.36 \%$ and 283 female students representing $54.63 \%$ were randomly selected from both public and private secondary schools in Ikeja Lagos were recruited into the study.

The Cochran method for sample size determination was used where 518 students were eligible to participate in the study and the sample population selected through a simple random sampling to collect the data.

Criteria for selecting the subjects were: i) Being students within secondary schools located within Ikeja Lagos and ii) Students with no reports of lower limb problems congenital or acquired.

However, students with visible known foot deformities, previous orthopedic corrective surgery were excluded.

The questionnaire design was done following the study's objective protocol:

The research questionnaire captured: i) Demographic characteristics of the respondents (age, gender, ethnicity); ii) General anthropometric measurements; iii) Foot Posture Index Assessment Scoring; iv) The measurements were recorded in the metric unit centimeters $(\mathrm{cm})$.

Anthropometers used for this survey include the following: i) Modified Harris Matts and Ink Pad; ii) Stadiometer (Field Type); iii) Tape rule; iv) Metal rule; v) Weighing scale.

All measurements were on a metric scale.

For each respondent, anthropometric measurements were collected and physically recorded into data tables before conversion to electronic data for statistical analysis.

Ikeja, Lagos was selected due to its cosmopolitan nature, which is home to diverse varieties of ethnic groups in Nigeria (both minority and majority), and being the hub of commercial activity within Lagos State. The demography elicited in the results is thus seen as reflective and representative of the Nigerian population.

The data collected was analyzed using STATA software version 14.0 and the results of the continuous measurements were presented on Mean \pm SD (Min-Max) while results on categorical measurements were presented in Number (\%). The relationship of the study group with BMI, Foot Posture Index, and Age distribution was done using Descriptive statistical analysis. The Chi-square test for assessing the statistical significance of associations at the 5\% level was also ascertained.

\section{Study consent and ethics approval}

This study was carried out in line with the recommendations of the Declaration of Helsinki with written informed consent/assent was obtained from all subjects. The study protocol was approved by the Lagos State University Health Research Ethics Committee (LRC/ 06/10/1438). Written permission to conduct the study was obtained from the Lagos State Government through the Ministry of Education District VI, Ideal Primary School, C/O, Apapa-Oshodi Express Way, Lagos. Written informed consent/assent was obtained having provided necessary identification and study documentation to each school for students, parents, and school administration before the enrolment of their students to participate in the study.

\section{Results}

\section{BMI of the study population}

Table 1 presents the study population of students' BMI as relates to the different age groups $(<12$ years, $12-17$ years, and 18 years). It was revealed that $49.66 \%$ of students within the age group of $12-17$ years had normal BMI. Also, $77.78 \%$ of students

Table 1. Study population BMI of students regarding their age.

\begin{tabular}{|c|c|c|c|c|c|}
\hline Body Mass Index $\left(\mathrm{Kg} / \mathrm{m}^{2}\right)$ & $\begin{array}{l}<12 \text { years } \\
\text { Freq. }(\%)\end{array}$ & $\begin{array}{c}\text { Age } \\
12-17 \text { years } \\
\text { Freq. }(\%)\end{array}$ & $\begin{array}{c}18 \text { years } \\
\text { Freq. (\%) }\end{array}$ & Total & p \\
\hline Underweight & $33(89.19)$ & 193(43.37) & $6(16.67)$ & $232(44.79)$ & 0.000 \\
\hline Normal & $4(14.29)$ & 221(49.66) & $28(77.78)$ & 253(49.71) & 0.000 \\
\hline Overweight & $0(0.00)$ & $22(4.94)$ & $1(2.78)$ & $23(4.52)$ & 0.000 \\
\hline Obese & $0(0.00)$ & $9(2.02)$ & $1(2.78)$ & $10(1.96)$ & 0.000 \\
\hline Total & $37(100)$ & $445(100)$ & $36(100)$ & $518(100)$ & 0.000 \\
\hline
\end{tabular}

Pearson $\operatorname{chi}^{2}(6)=32.4345 ;$ * Significant at $p<0.05$

Table 2. Study population BMI of students regarding gender.

\begin{tabular}{lcccc} 
& Total $(\mathrm{n}=5 \mathbf{1 8})$ Freq. (\%) & Male Freq. (\%) & Female Freq. (\%) & \\
Underweight $(<18.5)$ & $232(44.79)$ & $124(52.77)$ & $108(38.16)$ & $0.000^{*}$ \\
Normal $(18.5-25)$ & $255(50.09)$ & $103(44.01)$ & $152(55.27)$ & $0.000^{*}$ \\
\hline Overweight $(25-30)$ & $23(4.51)$ & $7(2.99)$ & $16(5.82)$ & $0.000^{*}$ \\
Obese $(>30)$ & $8(2.14)$ & $1(0.43)$ & $7(2.55)$ & $0.000^{*}$ \\
\hline Total & $518(100)$ & $235(100)$ & $283(100)$ & $0.000^{*}$ \\
\hline
\end{tabular}


in the age group of 18 years had normal BMI. On the other hand, $89.19 \%$ of students with the age range $<12$ years were underweight as against a small fraction constituting $14 \%$ that had normal BMI. The result of the Pearson chi-square, $\mathrm{X}^{2}=32.4345, \mathrm{p}=0.000$ shows that there is a statistically significant association between the study population BMI of student's cohort and their age.

The demographic data of Body Mass Index, BMI $\left(\mathrm{kg} / \mathrm{m}^{2}\right)$ distribution of secondary school students in groups of male and female indicated in the study is shown in Table 2. The test revealed that $52.77 \%$ (underweight) of males were in the BMI $<18.5$ while $44.02 \%$ (normal weight) were in the BMI range of $18.5-25$ with the remaining fraction of the population $(3.42 \%)$ exhibiting BMI $>25$ (Overweight). In the females, $38.16 \%$ were in the BMI $<18.5$ (underweight) and 55.27\% were in the range of 18.5 - 25 (normal weight). Those in the overweight/obese category (BMI $>25)$ constituted the remaining $8.37 \%$. The result of the Pearson chi-square $X^{2}=19.8967, \mathrm{p}=0.00$ shows that there was a statistically significant association between the gender groups and BMI. There was also a statistically significant association between different age groups and gender at $\mathrm{p}<0.046$. However, there was no statistically significant association between School type $(\mathrm{p}=0.703)$, class category $(\mathrm{p}=0.282)$.

\section{Body mass index on foot posture alignment}

The result of the histogram plot as presented in Figure 1 shows that Body Mass Index (BMI) distribution amongst students for the varying foot alignment is not normally distributed but instead skewed to the right.

Kruskal-Wallis $\mathrm{H}$ test (Table 3 ) assumes no normality in the distribution; hence it was conducted to determine if the BMI of students was statistically different for the three (3) groups of foot alignment; Pronated (51), Normal posture (432), and Supinated (35). The test showed that there was no statistically significant difference in BMI between the three (3) groups of student's foot alignment in resting gait; Pearson chi-square, $X^{2}(2)=4.575$, $\mathrm{p}=0.1015$.

\section{Standard deviation error bars on mean body mass index}

The result of the bar chart as presented in Figure 2 shows that the BMI distribution amongst students (male and female) for the varying foot posture alignment/resting gait had an overlap in the standard deviation error bars, hence the BMI of students is not statistically different for the three groups of foot alignment; normal, pronated and Supinated.

\section{Foot posture alignment Index (resting gait) distribution in males and females}

Foot Posture Index (FPI) is a revised clinical tool aimed at quantifying the degree to which a foot is aligned and can be considered to be in a pronated, supinated, or normal position. The distribution of the left foot posture alignment according to the FPI scale is listed in Table 4 below. In all, $82.13 \%$ of male students and $84.40 \%$ of female students showed normal the foot. $10.64 \%$ and $9.22 \%$ of the male and female students respectively confirmed slight foot pronation while $7.23 \%$ of male students and $6.38 \%$ of female students showed slightly supinated feet. The result of the $\mathrm{X}^{2}=0.4778, \mathrm{p}=0.788$ shows that there was no statistically significant association between gender and left foot alignment; that is both male and female students in the majority equally presented a normal left foot alignment.

The distribution of the right foot posture alignment according to the FPI scale is listed in Table 4 . In all, $78.30 \%$ of male students and $75.09 \%$ of female students showed normal foot $15.32 \%$ and $18.51 \%$ of the male and female students respectively confirmed slight foot pronation while $6.38 \%$ of male students and $6.41 \%$ of female students showed slightly supinated feet. The result of the $\mathrm{X}^{2}=0.9340, \mathrm{p}=0.627$ shows that there was no statistically significant association between gender and the right foot alignment; that is both male and female students in the majority equally presented a normal right foot alignment.

Table 3. Kruskal-Wallis equality-of-populations rank test.

\begin{tabular}{lccc} 
Foot Posture Alignment (Resting Gait) & Freq. & Percentage (\%) & Rank Sum \\
Pronated & 51 & 9.86 & 13876.50 \\
Normal & 432 & 83.40 & 106863.00 \\
\hline Supinated & 35 & 6.77 & 10588.50 \\
\hline Significant at $p<0.05$ Pearson Chi Square, $\chi^{2}=0.564 ; \mathrm{df}=2$ & & 0.1015 & 0.1015
\end{tabular}

*Significant at $\mathrm{p}<0.05$ Pearson Chi Square, $\chi^{2}=0.564 ; \mathrm{df}=2$

Table 4. Total FPI score distribution in girls and boys studied.

\begin{tabular}{|c|c|c|c|c|}
\hline Foot Alignment & $\begin{array}{c}\text { Gender } \\
\text { Males Freq. (\%) }\end{array}$ & Females Freq. (\%) & Total (\%) & $\mathbf{p}$ \\
\hline $\begin{array}{l}\text { Left Foot } \\
\text { Normal } \\
\text { Pronated } \\
\text { Supinated } \\
\text { Total }\end{array}$ & $\begin{array}{c}193(82.13) \\
25(10.64) \\
17(7.23) \\
235(100)\end{array}$ & $\begin{array}{c}239(84.40) \\
26(9.22) \\
18(6.38) \\
283(100)\end{array}$ & $\begin{array}{c}432(83.37) \\
51(9.86) \\
35(6.77) \\
518(100)\end{array}$ & $\begin{array}{l}0.788 \\
0.788 \\
0.788 \\
0.788\end{array}$ \\
\hline $\begin{array}{l}\text { Right Foot } \\
\text { Normal } \\
\text { Pronated } \\
\text { Supinated } \\
\text { Total }\end{array}$ & $\begin{array}{c}184(78.30) \\
36(15.32) \\
15(6.38) \\
235(100)\end{array}$ & $\begin{array}{c}213(75.09) \\
52(18.51) \\
18(6.41) \\
283(100)\end{array}$ & $\begin{array}{c}397(76.64) \\
88(17.05) \\
33(6.40) \\
518(100)\end{array}$ & $\begin{array}{l}0.627 \\
0.627 \\
0.627 \\
0.627\end{array}$ \\
\hline
\end{tabular}

Left foot - Pearson $\operatorname{chi}^{2}(2)=0.4778 *$ Significant at $p<0.05$. Right foot - Pearson chi ${ }^{2}(2)=0.9340 *$ Significant at $p<0.05$ 


\section{Discussion}

The objective of this investigation was to determine how the BMI of secondary school students in a cosmopolitan city would affect their FPA in a resting gait. The investigation, which was on students of age range $10-18$ years, indicated variability in BMI levels. BMI is arguably the most frequently used clinical parameter for body size assessment. The result of this research shows that most of the students of age cohort less than 12 years were underweight $(89.19 \%)$. Although the underweight incidence decreased considerably (43.37\%) amongst students of age cohort 12-17 years, the number nonetheless was still significant. However, for students, 18 years of age, only $16.67 \%$ of the population exhibited underweight. Using the statistics of Pearson chi-square analysis, age-dependent variability of BMI $\left(X^{2}=32.4345, p=0.000\right)$ was established amongst the study population. It further validated reports that showed BMI to be age-dependent. ${ }^{15,19}$ In all, the anthropometric measures show a tendency towards low BMI values in the population investigated. The foot posture index status however revealed that $83.40 \%$ of students had normal posture, $9.86 \%$ with pronation, and $6.77 \%$ with supination. On the balance greater proportion of the students' population exhibited normal foot alignment. Indeed, using the Kruskal-Wallis $\mathrm{H}$ test for the analysis, no probable relationship was established between BMI and FPI $\left(X^{2}(2)=4.575, p=0.1015\right)$ in resting gait. Also, the observed overlap in the standard deviation error bars for the varying foot posture alignment/resting gait further buttressed that the BMI of students was not statistically different for the three groups of foot alignment. These findings confirm earlier reports. ${ }^{14,15,20}$

The histogram for the BMI distribution curve for the varying foot alignment of the students was not normally distributed but skewed to the right. The justification for the observed pattern was obvious from the students' weight status descriptive statistics which indicates that only $6.48 \%$ of the students exhibited BMI $>25$ (overweight) in the study population while the greater proportion of the age cohort exhibited BMI $<25$. The weight disparity which tended towards the lower weight status triggered the skewness of the curve tail to the right. By implication, it depicts that the "bell" of the curve drifted to the opposite side (the left side), which was a measure of the high density of students with low BMI levels. The skewed mode of BMI distribution was however not restrictive but shows a sequence of consistency in the three varying students' foot alignment. The apparent high incidence of low BMI values amongst the students' population was a product of several factors. Apart from the inherent genetic predisposition, there was the more encompassing environmental impact factor. Most of the students below the age of 12 years in this study were first-year secondary school students. Interestingly they constituted the greater population of the underweight $(89.19 \%)$ students with BMI $<18.5$. Obviously, at this stage, the challenge of adjusting to secondary school life more often coincides with important social, emotional, and psychological changes that negatively impact wellbeing. It has been asserted that more often when students change school or class there is the need to adjust to the new surroundings. ${ }^{21}$ However, it was probably too that a significant number of the students were malnourished because cumulatively $44.79 \%$ of the entire students' population were underweight. This might be predictable in the current circumstances of a high rate of inflation in the Nigerian economy that has driven many families to lower socioeconomic strata in which case a significant proportion may well have resorted to an unhealthy dietary lifestyle. This allusion is in tandem with the earlier reports which stressed that adverse economic condition influences diet and subsequently, the health status of the citizenry. ${ }^{22-24}$ It has also been reported that being underweight in adolescence is associated with low socio-economic status. ${ }^{25}$

The effect of age and gender on FPA has been reported. ${ }^{20}$ However, separate postural status on the two legs has not been adequately highlighted amongst the two genders. In this study, the postural status of the left and right leg was discretely assessed in both sexes. The findings indicate that $82.13 \%$ of male students and $84.40 \%$ of female students exhibited normal left foot posture in resting gait whereas $10.64 \%$ and $9.22 \%$ of the male and female students respectively had slightly pronated left foot posture. The distribution on the right foot indicates the normal posture of $78.30 \%$ in male students and $75.09 \%$ in female students while $15.32 \%$ and $18.51 \%$ of the male and female students respectively confirmed right foot pronation. Foot supination however was concurrently least occurring in both genders' feet. Pearson chi-square analysis shows no significant $\left(\mathrm{X}^{2}=0.4778, \mathrm{p}=0.788\right)$ association between gender and left foot alignment. Similarly, no significant $\left(\mathrm{X}^{2}=0.9340, \mathrm{p}=0.627\right)$ association was established between gender and right foot alignment. It was therefore evident from this analysis that foot posture score distributions between the male and the female students did not exhibit remarkable difference thus con-

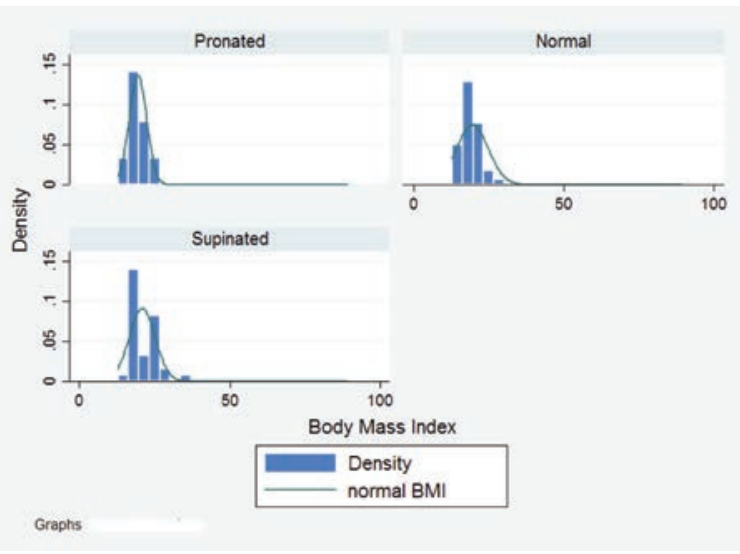

Figure 1. Histogram showing the distribution Body Mass Index on Foot Posture Alignment.

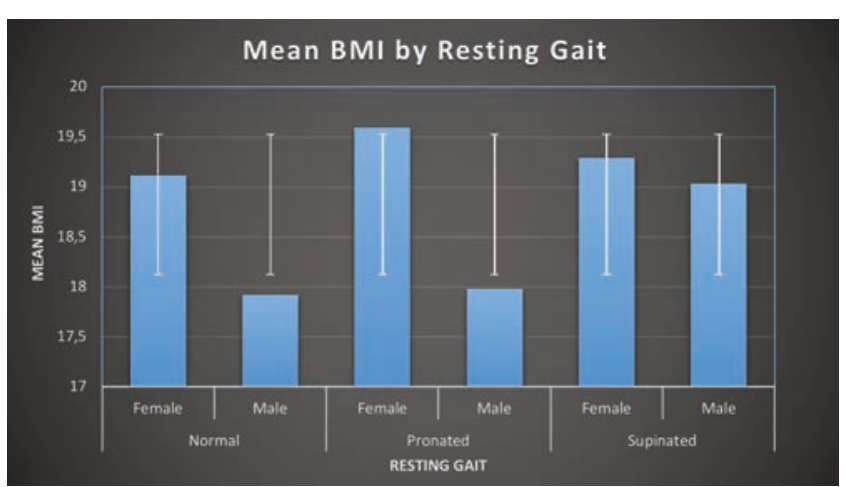

Figure 2. Standard deviation error bars showing the mean Body Mass Index (BMI) by resting gait. 
firming the reports of previous studies. ${ }^{14,20}$

The analysis of foot alignment measures amongst the two genders displays more unevenness in both legs of females' foot alignment. The disparity in FPA in females was more evident in the normal and pronated postural status. According to this study, the females recorded a total of $84.40 \%$ normal left foot alignment and $75.09 \%$ normal right foot alignment postures while in pronation, $9.22 \%$ for left foot posture, and $18.51 \%$ for right foot posture. The cause of the significant difference remains vague. Nevertheless, it has been made obvious that statistically determined reference ranges for postural variations such as in standing foot position are inherently wide, consequently, it should be used as a general guide only in interpreting FPI scores in a clinical context. ${ }^{14}$

\section{Conclusions}

It was established in this study that BMI has no influence on FPI in resting gait. The high incidence of underweight amongst the entire students' population suggests the likely influence of socioeconomic factors. However, amongst the two genders, the females display more unevenness in foot alignment between the two legs.

\section{References}

1. Levinger P, Lenz HB, Fotoohabaddi R, et al. Foot posture in people with medial compartment knee osteoarthritis. J Foot Ankle Res 2010;3:1-8.

2. AlAbdulwahab SS, Kachanathu SJ. Effects of body mass index on foot posture alignment and core stability in a healthy adult population. J Exerc Rehabil 2016;12:182-7.

3. Ganu SS, Panhale V. Effect of Obesity on Arch Index in Young Adults. Online J Health Allied Sc 2013;11:1-3.

4. Butterworth PA, Urquhart DM, Landorf KB, et al. Foot posture, the range of motion and plantar pressure characteristics in obese and nonobese individuals. Gait Posture 2015;41:465-9.

5. Shariff SM, Manaharan T, Shariff AA, Merican AF. Evaluation of foot arch in adult women: comparison between five different footprint parameters. Sains Malaysiana 2017;46:1839-48.

6. Puepet FH, Zoakah AI, Chuhwak EK. Prevalence of overweight and obesity among urban Nigeria adults in Jos. Highland Med Res J 2002;1:13-6.

7. Adienbo OM, Hart VO, Oyeyemi WA. High prevalence of obesity among indigenous residents of a Nigerian ethnic group: The Kalabaris in the Niger delta region of South South Nigeria. Greener J Med Sci 2012;2:152-6.

8. Ulasi II, Ijoma CK, Onodugo OD, et al. Towards prevention of chronic kidney disease in Nigeria: A community-based survey in southeast Nigeria. Kidney Int suppl 2013;3:195-201.

9. Gezawa ID, Puepet FH, Mubi BM, et al. Prevalence of overweight and obesity in Maiduguri, North-Eastern Nigeria. Niger J Med 2013;22:171-4.
10. Hue O, Simoneau M, Marcotte J, et al. Body weight is a strong predictor of postural stability. Gait Posture 2007;26:32-8.

11. Steele JR, Riddiford-Harland DL, Mickle KJ. Excessive weight-bearing compromises foot structure and function across the lifespan. In Gefen A, Benayahu D (eds), The Mechanobiology of Obesity and Related Diseases, Springer: New York, 2015; p. 149-79.

12. Krishna HS, Basheer R, Jazeel KB, et al. Correlation of body mass index with navicular height in young's. Inter $\mathrm{J}$ Information Res Rev 2018;5:5659-62.

13. Lin CJ, Lin SC, Huang W, et al. Physiological knock-knee in preschool children: prevalence, correlating factors, gait analysis, and clinical significance. J Pediatr Orthop 1999;19:650-4.

14. Redmond AC, Crane YZ, Menz HB. Normative values for the Foot Posture Index. J Foot and Ankle Res 2008;1:6.

15. Arthi G, Sadeesh T, Raghuram K. Effect of height, weight and BMI on foot postures of young adult individual. J Clin Diagnos Res 2018;12:AC06-8.

16. Lobstein T, Baur I, Uauy R, IASO International Obesity Task Force. Obesity in children and young people - A crisis in Public Health. Obes Rev 2004:5:S4-S85.

17. Saltzman CL, Nawoczenski DA, Talbot KD. Measurement of the medial longitudinal arch. Arch Phys Med Rehabil 1995;76:45-9.

18. Hernandez AJ, Kimura LK, Laraya MHF, Favaro E. Calculation of staheli's plantar arch index and prevalence of flat feet: a study with 100 children aged 5-9 years. Acta Ortop Bras 2007;15:68-71.

19. Evans AM, Karimi L. The relationship between paediatric foot posture and body mass index: do heavier children really have flatter feet? J Foot Ankle Res 2015;8:46.

20. Staheli LT, Chew DE, Corbett M. The longitudinal arch. A survey of eight hundred and eighty-two feet in normal children and adults. J Bone Joint Surg 1987;69A:426-8.

21. West P, Sweeting H, Young R. Transition matters: pupils' experiences of the primary-secondary school transition in the West of Scotland and consequences for well-being and attainment. J Res Papers Edu 2010;25:21-50.

22. Antentas JM, Vivas E. Impact of the economic crisis on the right to a healthy diet. SESPAS report 2014. Gac Sanit 2014;28:58e61.

23. Bonaccio M, Di Castelnuovo A, Bonanni A, et al. Decline of the Mediterranean diet at a time of economic crisis. Results from the Moli-sani study. Nutr Metab Cardiovasc Dis 2014;24:853e60.

24. Marra M, Migliardi A, Costa G. Health inequalities and nutrition in Italy during crisis times. Epidemiol Prev 2015;39:322e31.

25. Grammatikopoulou MG, Poulimeneas D, Maraki MI, et al. Geographical distribution of simple and abdominal obesity among 17-year-old adolescents in Greece. Obes Med 2016; $2: 31 \mathrm{e} 6$. 